\title{
Evolutionary analysis of genes coding for Cysteine-Rlch Secretory Proteins (CRISPs) in mammals
}

\author{
Lena Arévalo ${ }^{1,2}$, Nicolás G. Brukman³ ${ }^{3}$ Patricia S. Cuasnicú ${ }^{3}$ and Eduardo R. S. Roldan ${ }^{*}$
}

\begin{abstract}
Background: Cysteine-RIch Secretory Proteins (CRISP) are expressed in the reproductive tract of mammalian males and are involved in fertilization and related processes. Due to their important role in sperm performance and sperm-egg interaction, these genes are likely to be exposed to strong selective pressures, including postcopulatory sexual selection and/or male-female coevolution. We here perform a comparative evolutionary analysis of Crisp genes in mammals. Currently, the nomenclature of CRISP genes is confusing, as a consequence of discrepancies between assignments of orthologs, particularly due to numbering of CRISP genes. This may generate problems when performing comparative evolutionary analyses of mammalian clades and species. To avoid such problems, we first carried out a study of possible orthologous relationships and putative origins of the known CRISP gene sequences. Furthermore, and with the aim to facilitate analyses, we here propose a different nomenclature for CRISP genes (EVAC1-4, "EVolutionarily-analyzed CRISP") to be used in an evolutionary context.
\end{abstract}

Results: We found differing selective pressures among Crisp genes. CRISP1/4 (EVAC1) and CRISP2 (EVAC2) orthologs are found across eutherian mammals and seem to be conserved in general, but show signs of positive selection in primate CRISP1/4 (EVAC1). Rodent Crisp1 (Evac3a) seems to evolve under a comparatively more relaxed constraint with positive selection on codon sites. Finally, murine Crisp3 (Evac4), which appears to be specific to the genus Mus, shows signs of possible positive selection. We further provide evidence for sexual selection on the sequence of one of these genes (Crisp 1/4) that, unlike others, is thought to be exclusively expressed in male reproductive tissues.

Conclusions: We found differing selective pressures among CRISP genes and sexual selection as a contributing factor in CRISP1/4 gene sequence evolution. Our evolutionary analysis of this unique set of genes contributes to a better understanding of Crisp function in particular and the influence of sexual selection on reproductive mechanisms in general.

Keywords: Sexual selection, CRISP, Mammals, Sperm, Fertilization

\section{Background}

Proteins of the reproductive system that affect male and female traits are thought to be targets of accelerated gene sequence evolution $[1,2]$. However, whereas this is generally true, evolutionary rates of reproductive proteins vary depending on their involvement in different reproductive

\footnotetext{
* Correspondence: roldane@mncn.csic.es

${ }^{1}$ Department of Biodiversity and Evolutionary Biology, Museo Nacional de Ciencias Naturales (CSIC), C/José Gutiérrez Abascal 2, 28006 Madrid, Spain Full list of author information is available at the end of the article
}

processes or localization of expression [3-5]. One of the main driving forces promoting sequence divergence is postcopulatory sexual selection, either in the form of sperm competition or as cryptic female choice, which can additionally increase sexual conflict and drive male-female co-evolution leading to rapid adaptation. Sperm competition occurs when females mate promiscuously, and ejaculates of rival males compete for fertilizations. This leads to adaptations improving sperm performance; it may also

C C The Author(s). 2020 Open Access This article is licensed under a Creative Commons Attribution 4.0 International License, which permits use, sharing, adaptation, distribution and reproduction in any medium or format, as long as you give appropriate credit to the original author(s) and the source, provide a link to the Creative Commons licence, and indicate if changes were made. The images or other third party material in this article are included in the article's Creative Commons licence, unless indicated otherwise in a credit line to the material. If material is not included in the article's Creative Commons licence and your intended use is not permitted by statutory regulation or exceeds the permitted use, you will need to obtain permission directly from the copyright holder. To view a copy of this licence, visit http://creativecommons.org/licenses/by/4.0/ The Creative Commons Public Domain Dedication waiver (http://creativecommons.org/publicdomain/zero/1.0/) applies to the data made available in this article, unless otherwise stated in a credit line to the data. 
intensify cryptic female choice and sexual conflict due to greater potential for selection [6].

The effect of postcopulatory sexual selection on evolutionary rates of reproductive proteins has been studied widely. Yet, it has been difficult to detect clear signals of sexual selection and only a small set of studies have found significant evidence by using correlational approaches. For example, the evolutionary rates of the coding sequences of seminal fluid proteins SEMG2 and SVS2, and of proteins expressed on the sperm surface (ADAM2 and ADAM18) and the acrosome (ZAN and SPAM1) have been found to be positively correlated with post-copulatory sexual selection in primates [7-11]. Other studies have reported negative correlations of sexual selection with evolutionary rates, such as in seminal fluid proteins in butterflies [12] and in sperm nuclear protamine 1 and protamine 2 in rodents [13-15]. Proteins found on the sperm surface [16] and those with roles in sperm motility and sperm-egg interaction [5] have been found to have particularly high evolutionary rates.

The mammalian spermatozoon is a very complex, polarized cell that needs to undergo a series of processes such as maturation in the epididymis and capacitation in the female tract in order to be able to reach, recognize and fertilize the egg in the oviduct. Spermatozoa carry numerous proteins involved in the acquisition of their fertilizing ability as well as in gamete interaction [3]. The Cysteine-RIch Secretory Protein (CRISP) family is of specific interest because it is involved in several of these processes and, therefore, is a likely target for sexual selection-driven evolution of gene sequences. Members of the CRISP family are mainly expressed in the mammalian male reproductive tract [17] and in the venoms of snakes [18]. Two major functional domains are present in all CRISPs: the PR-1 (or CAP) domain, which is thought to be involved in cell-cell adhesion, i.e., association between germ and Sertoli cells and sperm-egg fusion $[17,19,20]$, and the Cysteine-Rich Domain (CRD), containing 16 conserved cysteine residues, with the capacity to regulate ion channels $[17,21]$. The CRISP family, together with the Antigen-5 and the Pathogenesis related-1 proteins, form the CAP superfamily of proteins found in a wide range of organisms (bacteria, yeast, fungi, insects, plants and mammals, including human). The tertiary structure of CAP proteins shows a remarkable conservation despite often low overall identity and significant phylogenetic distance between organisms, suggesting that these proteins may be involved in common and essential biological processes [17]. A recent study investigating the evolutionary history of CAP proteins showed that the exon structure and borders of CRISP genes are remarkably conserved among vertebrates as compared to invertebrate CAP proteins [22].
Mammalian CRISPs are highly expressed during sperm cell development and maturation as well as during fertilization. Most mammals have three CRISP genes while in mice four CRISP members have been described: CRISP1, a mainly epididymal protein [23], CRISP2 [24], highly expressed in the testes, CRISP3 [25], which is widely distributed in reproductive and non reproductive organs, and CRISP4 which is mainly synthetized in the epididymis [26]. In the past few years, the use of in vitro approaches [20, 27-29] and knockout studies aimed at characterizing CRISPs [30-34] revealed the involvement of these proteins in different stages of the fertilization process (see review in [35]). Rodent CRISP1 binds to the sperm plasma membrane during epididymal maturation and is associated with both sperm-zona pellucida binding and gamete membrane fusion through the interaction of the protein with complementary sites in the egg [27, 36, 37]. Whereas evidence supports that the ability of CRISP1 to interact with the egg plasma membrane during gamete fusion resides in a region of only 12 amino acids within the PR-1 (CAP) domain that corresponds to one of the signatures of the CRISP family [20], the interaction of the protein with the zona pellucida does not reside in any specific region of the PR-1 domain but rather depends on the entire conformation of the molecule [29]. Interestingly, recent results showed that CRISP1 is also expressed in the cumulus cells that surround the egg and plays a role in fertilization [38]. Additionally, it has been revealed that CRISP1 has the ability to regulate CatSper [38], the principal sperm $\mathrm{Ca}^{2+}$ channel involved in the development of hyperactivation and essential for male fertility $[39,40]$. Based on previous reports showing the ion regulatory activity of the CRD, it is likely that CRISP1 regulates CatSper through this domain [38]. Similar to rodent CRISP1, CRISP2 seems to play a role in gamete fusion $[28,29]$. Recent knockout studies showing evidence for its involvement in hyperactivation development during capacitation and in both cumulus and zona pellucida penetration further strengthened this hypothesis [31]. Whereas no roles for CRISP3 in sperm function have been reported so far, the generation of CRISP4 knockout mice supports the involvement of this epididymal protein in fertilization [34, 36].

The sequences of mammalian CRISP genes were found to be conserved when compared to CRISP genes of snake venoms [41]. Yet, despite the prominent role of mammalian CRISPs in sperm capacitation and fertilization, comparative selective pressures and the role of sexual selection on CRISP gene sequence divergence has been scarcely addressed [5, 41, 42]. During this study, we aimed to fill this gap by examining patterns of selective pressures in mammalian clades and the role of sexual selection in the evolution of CRISP gene sequences. Currently, the nomenclature 
of the CRISP genes is somewhat confusing, as a consequence of discrepancies between assignments of orthologs, particularly due to numbering of CRISP genes [17], and this may generate problems when performing comparative evolutionary analyses between mammalian clades and species. Therefore, we first carried out an analysis of possible orthologous relationships and putative origins of the known CRISP gene sequences and, thus, propose a different nomenclature for CRISP genes when analyzed in an evolutionary context. Secondly, we analyzed selective pressures and sexual selection driving CRISP evolutionary rates for a relevant subset of CRISP genes. Even though CRISP gene sequences seem to be conserved in general [41], based on their involvement in sperm capacitation and sperm-egg interaction, we expected accelerated evolutionary rates concentrated on functionally relevant codon sites and regions. However, the main goal of this study was the general characterization of selection pressures (conservation, relaxation and/or positive selection) on Crisp gene sequences in mammalian clades. Additionally, we expected gene sequence divergence to be driven by sexual selection including sperm competition, cryptic female choice and/or malefemale coevolution.

\section{Results}

\section{CRISP nomenclature and sequence analysis}

In order to perform comparative evolutionary studies between clades and species for the different CRISP genes we first needed an analysis of sequence identity, location and possible origin of the different CRISP genes. Current nomenclature of CRISP genes can lead to confusion when comparing species and clades because of discrepancies in the numbering of CRISP genes (Fig. 1a). To avoid misinterpretations, we considered possible origins of CRISP genes during their evolutionary history based on gene sequence identity scores according to NCBI Blast [43], the existence or non-existence of specific CRISP orthologs in selected species, and information by Nolan et al. [45] and Vadnais et al. [44] (Fig. 1a). We also calculated a CRISP gene tree based on the sequence data used in this study (Additional files 1 and 2). Based on this information, we here propose a different nomenclature to be used in the evolutionary analyses of CRISP genes which are hereafter designed as "EVolutionarilyanalyzed CRISPs" (=EVAC) (see Fig. 1b for details of nomenclature). In addition, we present a tentative representation of the origins of EVAC duplication events (Fig. 2). The numbering of EVAC genes employed here follows the proposed/possible sequence of evolutionary history from the most ancestral gene to the most recently arisen duplication. It should be borne in mind that this analysis is not exhaustive and has as its main goal attaining sufficient confidence in gene relationships so as to perform a comparative evolutionary analysis.

\section{Selective pressures on EVAC1 and EVAC2 Across mammals}

EVAC1 and EVAC2, which are found across mammals (Fig. 1b), were tested for the general mode of selection acting upon them. To obtain the selective pressure acting on the whole sequence across all mammals, we calculated the evolutionary rate $(\omega)$ for the whole tree on the whole sequence (Codeml (PAML4) model M0, as explained in Materials and Methods). The evolutionary rate calculated across mammals in model M0 was EVAC1: $\omega=0.48$ and EVAC2: $\omega=0.33$.

\section{Comparison of selective pressures between mammalian clades}

Clade-specific analyses were performed on clades for which sequence data of at least 6 species were available (EVAC1: Primates, Rodentia, Carnivora, Cetartiodactyla; EVAC2: Primates, Rodentia, Chiroptera, Cetartiodactyla). To assess the comparative selective pressures for the entire sequence and selective pressures on codon sites, we employed branch analysis and branch-site analysis (see Materials and Methods), marking the clade of interest as foreground against the remaining species as background.

The branch analysis for EVAC1 comparing clades suggests conserved selective constraint on all clades (LRT MCfixed vs MC significant, $\omega$ is significantly lower than 1) The selective constraint seems to be stronger in rodents and Cetartiodactyla (LRT M0 vs MC significant, MC $\omega$ considered, MC $\omega<$ M0 $\omega)$ (Table 1). The branch-site test for EVAC1 showed positive selection on 2 codon sites for primates (BSfixed vs BS significant, 222-I, 235-I) (Table 1).

The branch analysis for EVAC2 also suggests conserved selective constraint on all clades (LRT MCfixed vs MC significant, $\omega$ is significantly lower than 1 ). Here, in Chiroptera a comparatively more relaxed constraint is detected (LRT M0 vs MC significant, MC $\omega$ considered, MC $\omega>$ M0 $\omega$ ) (Table 2). The branch-site test for EVAC2 showed no signs of positive selection on codon sites in either clade (BSfixed vs BS non significant) (Table 2).

\section{Selective pressures on EVAC3 Across rodents}

Evac3a seems to be the ortholog of human EVAC3 (Fig. 1b), although it seems to have evolved more rapidly in rodents leading to a lower sequence identity than expected when compared to non-rodent species or other CRISP family members (see Fig. 1a,b). We therefore confined our comparative analysis to rodent Evac3a. The alignment and phylogenetic tree used in evolutionary analyses is shown in additional files 4 and 5 .

To obtain the selective pressure acting on Evac3a sequence across all rodents, we calculated the evolutionary rate $(\omega)$ for the whole tree (Codeml (PAML4) model M0 


\section{COMPARATIVE VIEW OF CRISP GENES}

A - current nomenclature

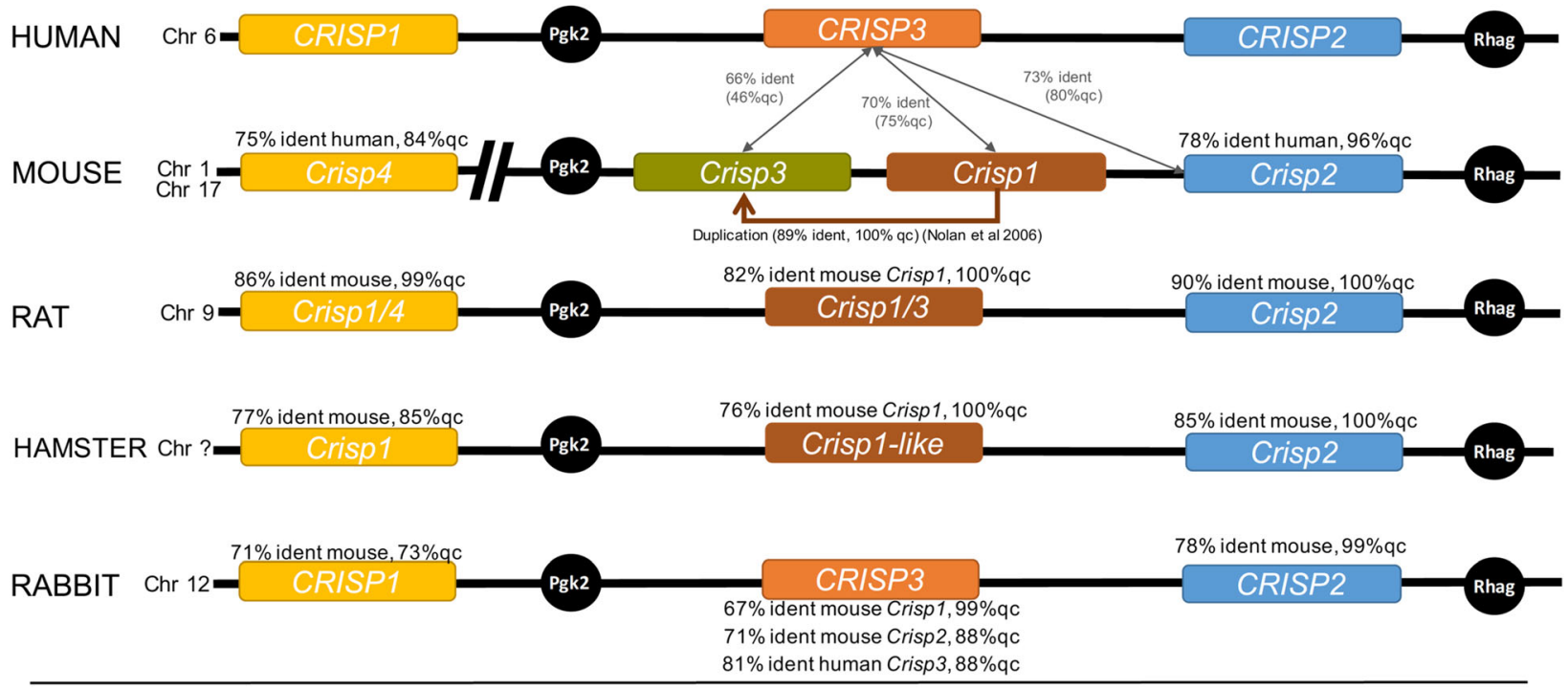

B - proposed nomenclature
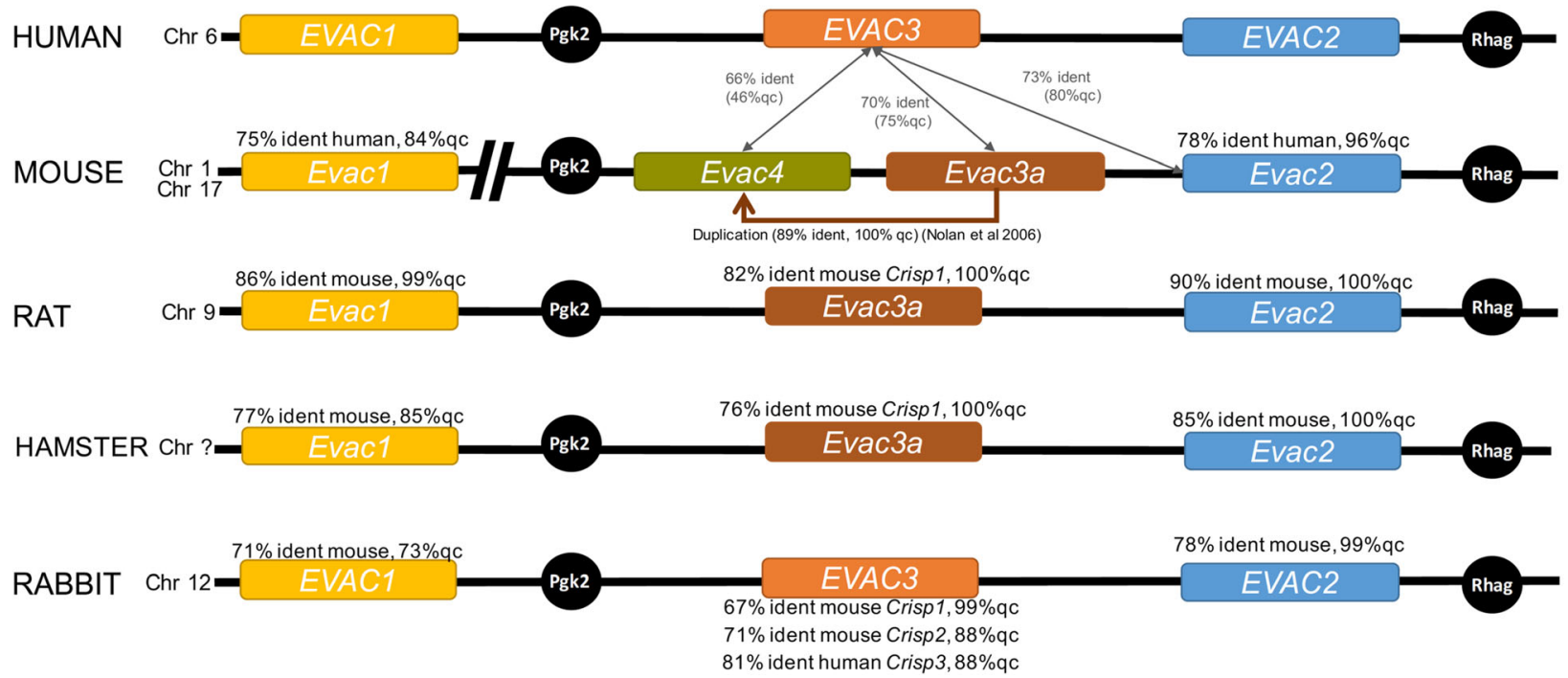

Fig. 1 CRISP nomenclature and proposed evolutionary history. Information on chromosomal location, nomenclature and sequence identity of representative species is given. Sequence identity taken from NCBI nucleotide BLAST analyses using the genes coding sequences (Altschul et al. [43]), information available in GenBank databases and literature is included. (A) Current nomenclature. (B) Proposed nomenclature for comparative evolutionary analysis. Ident = sequence identity, qc = query cover. Coloring indicates orthology. In part modified from Vadnais et al. [44]

as explained in Materials and Methods). The evolutionary rate calculated across rodents in model M0 was $\omega=$ 0.53 (Table 3 ). We additionally performed a site-analysis to determine if specific codon sites are positively selected across rodents. Two sites show a trend towards positive selection (M7 vs M8 significant, M1a vs M2a non significant, 64-G, 73-T) (Table 3).
Comparison of selective pressures between rodent species Lineage-specific analyses were performed on species for which sequence data were available (Cricetulus griseus, Marmota marmota, Mesocricetus auratus, Microtus ochrogaster, Mus musculus, Nannospalax galili, Peromyscus maniculatus, Rattus norvegicus). Similar to the analysis of EVAC1 and EVAC2, we assessed the comparative 


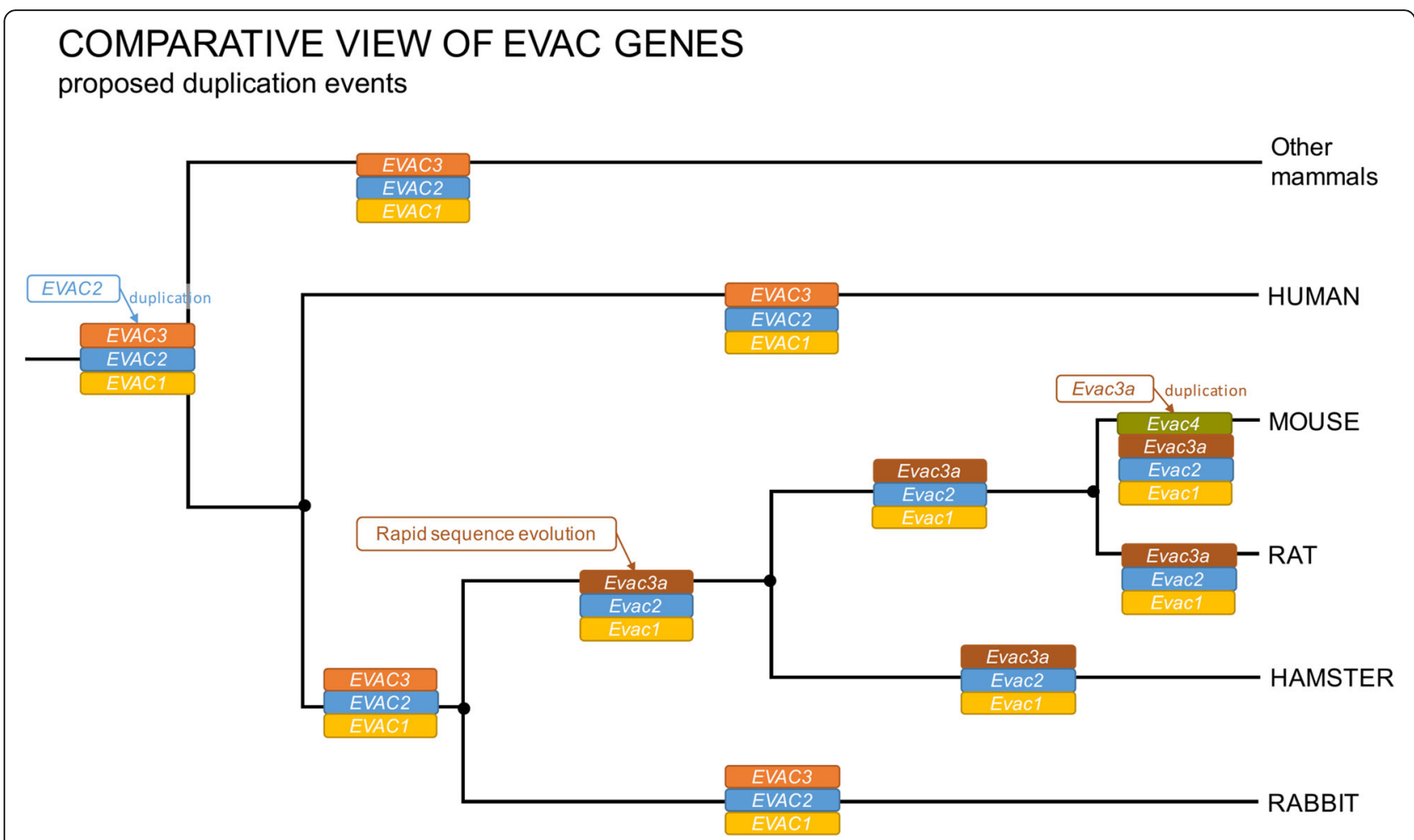

Fig. 2 Proposed CRISP (EVAC) duplication events. Phylogeny according to Lüke et al. [14, 15]

selective pressures for the entire sequence and on codon sites (branch analysis and the branch-site analysis), alternately marking a species branch as foreground against the remaining species as background.

The branch analysis for Evac3a comparing species suggests relaxed selective constraint (LRT MCfixed vs $\mathrm{MC}$ non significant, $\omega$ not significantly different from 1) on all species except Mesocricetus auratus for which a conserved constraint seems to be a better fit (LRT MCfixed vs MC significant, $\omega$ is significantly lower than 1) (Table 3). The branch-site test for Evac3a showed positive selection on codon sites for Marmota marmota (BSfixed vs BS significant, 35-E,
161-Y) and Microtus ochrogaster (BSfixed vs BS significant, 49-S, 225-K) (Table 3).

\section{Selective pressures on Evac4}

Based on extensive research in GenBank and PhylomeDB databases and NCBI Blast analyses [43], we propose that the Evac4 gene is a recent duplication present in the genus Mus. We did not expect any major differences in coding sequences between Mus species due to the high sequence identity between closely related species. In order to look for differences between species and sub-species in their coding sequence, we gathered Evac4 gene sequences from the mouse wild-derived

Table 1 Summary of results for EVAC1 branch analysis and branchsite analysis for mammalian clades

\begin{tabular}{|c|c|c|c|c|c|c|c|c|c|c|c|}
\hline \multirow{2}{*}{$\begin{array}{l}\text { EVAC1 } \\
\text { Foreground } \\
\text { branches }\end{array}$} & \multicolumn{2}{|c|}{ LRTs for Branch Analysis } & \multicolumn{2}{|l|}{ LRTs for BS analysis } & \multicolumn{4}{|c|}{$\begin{array}{l}\text { Prop. of sites in } \omega \\
\text { classes (BS): }\end{array}$} & \multirow[b]{2}{*}{ PSS (BEB $p<0.05)$} & \multicolumn{2}{|l|}{ Interpretation } \\
\hline & $2 \Delta$ (MO-MC) & $2 \Delta$ (MCfixed-MC) & $2 \Delta$ (BSfixed-BS) & $\omega$ & 0 & 1 & $2 a$ & $2 b$ & & $\begin{array}{l}\text { Selection over } \\
\text { whole sequence }\end{array}$ & $\begin{array}{l}\text { Selection } \\
\text { on sites }\end{array}$ \\
\hline Primates & -1.12 & $-36.43^{(<0.01)}$ & $-9.03^{(0.04)}$ & 0.48 & 0.44 & 0.53 & 0.01 & 0.01 & 2221,235 I & conserved & positive \\
\hline Rodentia & $-20.69^{(<0.01)}$ & $-152.48^{(<0.01)}$ & 0.00 & 0.35 & 0.44 & 0.53 & 0.01 & 0.01 & - & conserved & no \\
\hline Carnivora & -2.87 & $-41.55^{(<0.01)}$ & -5.97 & 0.48 & 0.45 & 0.55 & 0.00 & 0.01 & - & conserved & no \\
\hline Cetartiodactyla & $-20.90^{(<0.01)}$ & $-98.98^{(<0.01)}$ & 0.01 & 0.29 & 0.45 & 0.55 & 0.00 & 0.00 & - & conserved & no \\
\hline
\end{tabular}

Significant LRTs indicated in bold with FDR-corrected $p$-value in superscript, $\omega=$ clade omega as calculated by branch analysis, if LRT of M0 versus MC significant MC $\omega$ is reported if LRT non significant M0 $\omega$ is reported. $\omega$ site classes: $0: 0<\omega<1$ for foreground and background branches, $1: \omega=1$ for foreground and background branches, 2a: $0<\omega<1$ for background and $\omega>1$ for foreground, $2 \mathrm{~b}: \omega=1$ for background and $\omega>1$ for foreground. Applied models are explained in material and methods $(\mathrm{MC}=$ Clade model, $\mathrm{BS}=$ Branch-site model). PSS Positively selected sites, BEB Bayes empirical Bayes 
Table 2 Summary of results for EVAC2 branch analysis and branchsite analysis for mammalian clades

\begin{tabular}{|c|c|c|c|c|c|c|c|c|c|c|c|}
\hline \multirow{2}{*}{$\begin{array}{l}\text { EVAC2 } \\
\text { Foreground } \\
\text { branches }\end{array}$} & \multicolumn{2}{|c|}{ LRTs for Branch Analysis } & \multicolumn{2}{|l|}{ LRTs for BS analysis } & \multicolumn{4}{|c|}{$\begin{array}{l}\text { Prop. of sites in } \omega \\
\text { classes (BS): }\end{array}$} & \multirow[b]{2}{*}{ PSS (BEB $p<0.05)$} & \multicolumn{2}{|l|}{ Interpretation } \\
\hline & $2 \Delta(\mathrm{MO}-\mathrm{MC})$ & $2 \Delta$ (MCfixed-MC) & $2 \Delta$ (BSfixed-BS) & $\omega$ & 0 & 1 & $2 a$ & $2 b$ & & $\begin{array}{l}\text { Selection over } \\
\text { whole sequence }\end{array}$ & $\begin{array}{l}\text { Selection } \\
\text { on sites }\end{array}$ \\
\hline Primates & -1.59 & $-63.97^{(<0.01)}$ & 0.02 & 0.33 & 0.61 & 0.39 & 0.00 & 0.00 & - & conserved & no \\
\hline Rodentia & -0.15 & $-148.19^{(<0.01)}$ & 0.00 & 0.33 & 0.58 & 0.35 & 0.05 & 0.03 & - & conserved & no \\
\hline Chiroptera & $-13.57^{(<0.01)}$ & $-5.47^{(0.05)}$ & 0.00 & 0.63 & 0.61 & 0.39 & 0.00 & 0.00 & - & conserved & no \\
\hline Cetartiodactyla & -4.82 & $-113.47^{(<0.01)}$ & 1.27 & 0.33 & 0.61 & 0.39 & 0.00 & 0.00 & - & conserved & no \\
\hline
\end{tabular}

Significant LRTs indicated in bold with FDR-corrected $p$-value in superscript, $\omega=$ clade omega as caculated by branch analysis, if LRT of M0 versus MC significant MC $\omega$ is reported if LRT non significant M0 $\omega$ is reported. $\omega$ site classes: $0: 0<\omega<1$ for foreground and background branches, 1: $\omega=1$ for foreground and background branches, 2a: $0<\omega<1$ for background and $\omega>1$ for foreground, $2 \mathrm{~b}: \omega=1$ for background and $\omega>1$ for foreground. Applied models are explained in material and methods ( $\mathrm{MC}=$ Clade model, $\mathrm{BS}=$ Branch-site model). PSS Positively selected sites, $B E B$ Bayes empirical Bayes

strain genome sequences available from the Sanger mouse genomes project [46]. Sequences from Mus musculus musculus strain PWK/PhJ, Mus musculus domesticus strain WSB/EiJ, Mus musculus castaneus strain CAST/EiJ, and Mus spretus strain SPRET/EiJ were trimmed to coding sequence and checked manually. The multiple sequence alignment was marked to visualize differences in coding sequence and amino acid changes. No differences were found between Mus musculus subspecies. A total of 14 nucleotide substitutions were found in the Mus spretus strain when compared to the Mus musculus strains. All except one of the nucleotide substitutions found in the Mus spretus sequence lead to amino acid changes $(89 \mathrm{~L}>\mathrm{I}, 107 \mathrm{~V}>\mathrm{A}, 114 \mathrm{Q}>\mathrm{E}, 149$ $\mathrm{Q}>\mathrm{K}, 169 \mathrm{R}>\mathrm{H}, 174 \mathrm{~L}>\mathrm{S}, 123 \mathrm{E}>\mathrm{T}, 230 \mathrm{G}>\mathrm{N})$. It is therefore likely that this gene evolves under positive selective pressure. Not enough sequence divergence was found to reliably perform Codeml analysis or to test for effects of sexual selection (Fig. 3).

\section{Sexual selection on EVACs}

To examine the possible effects of sexual selection on $E V A C$ evolutionary rates we employed COEVOL using relative testes mass as proxy for postcolulatory sexual selection (see Methods) [47]. We first performed the analysis using all mammalian species. Then we analyzed each mammalian clade separately using a clade specific alignment and phylogenetic tree. Our results showed a trend for a correlation between relative testes mass and EVAC1 $\omega$ in mammals (Fig. 4, Table 4). Within clades, we found a trend for a positive correlation between relative testes mass and Evac1 $\omega$ in rodents (Table 4). Correlations for the remaining clades were not significant. No correlation was found between relative testes mass and

Table 3 Summary of results for Evac3a branch analysis, site and branchsite analysis for mammalian clades

\begin{tabular}{|c|c|c|c|c|c|c|c|c|c|c|c|}
\hline Evac3a & \multicolumn{2}{|c|}{ LRTs for Branch Analysis } & \multicolumn{2}{|l|}{$\begin{array}{l}\text { LRTs for BS } \\
\text { analysis }\end{array}$} & \multicolumn{4}{|c|}{$\begin{array}{l}\text { Prop. of sites in } \omega \\
\text { classes (BS): }\end{array}$} & & \multicolumn{2}{|l|}{ Interpretation } \\
\hline Foreground branches & $2 \Delta(\mathrm{MO}-\mathrm{MC})$ & $2 \Delta$ (MCfixed-MC) & $2 \Delta$ (BSfixed-BS) & $\omega$ & 0 & 1 & $2 a$ & $2 b$ & $\begin{array}{l}\text { PSS (BEB } \\
p<0.05)\end{array}$ & $\begin{array}{l}\text { Selection over } \\
\text { whole sequence }\end{array}$ & $\begin{array}{l}\text { Selection } \\
\text { on sites }\end{array}$ \\
\hline Cricetulus griseus & -1.83 & -0.38 & -0.09 & 0.53 & 0.44 & 0.53 & 0.01 & 0.01 & - & relaxed & no \\
\hline Marmota marmota & $-7.18^{(0.04)}$ & -7.18 & $-8.07^{(0.03)}$ & 0.00 & 0.45 & 0.52 & 0.02 & 0.02 & $35 E, 161 Y$ & relaxed & positive \\
\hline Mesocricetus auratus & -0.44 & -4.51 & 0.00 & 0.53 & 0.46 & 0.54 & 0.00 & 0.00 & - & conserved & no \\
\hline Microtus ochrogaster & $-7.05^{(0.04)}$ & 0.00 & $-7.29^{(0.03)}$ & 1.00 & 0.43 & 0.45 & 0.06 & 0.06 & $49 \mathrm{~S}, 225 \mathrm{~K}$ & relaxed & positive \\
\hline Mus musculus & -0.12 & -2.02 & -1.67 & 0.53 & 0.45 & 0.54 & 0.00 & 0.01 & - & relaxed & no \\
\hline Nannospalax galili & -0.07 & -3.69 & $-7.30^{(0.03)}$ & 0.53 & 0.43 & 0.53 & 0.02 & 0.02 & $(8 \mathrm{~L})$ & relaxed & positive \\
\hline Peromyscus maniculatus & 0.00 & -2.41 & -5.03 & 0.53 & 0.45 & 0.54 & 0.01 & 0.01 & $187 \mathrm{~S}$ & relaxed & no \\
\hline \multirow[t]{2}{*}{ Rattus norvegicus } & -0.05 & -2.07 & 0.00 & 0.53 & 0.45 & 0.53 & 0.01 & 0.01 & - & relaxed & no \\
\hline & \multicolumn{4}{|c|}{ LRTs for Site Analysis } & \multicolumn{7}{|c|}{$\begin{array}{l}\text { prop of sites in } \omega \\
\text { classes (M2a): }\end{array}$} \\
\hline Over all branches & $2 \Delta(M 7-M 8)$ & $2 \Delta(\mathrm{M} 1 \mathrm{a}-\mathrm{M} 2 \mathrm{a})$ & & & 0 & 1 & 2 & & \multicolumn{2}{|c|}{$\begin{array}{l}\text { PSS (BEB } p<0.05 \text {, } \\
\text { both LRTs) }\end{array}$} & $\begin{array}{l}\text { Selection } \\
\text { on sites }\end{array}$ \\
\hline Rodentia & $-7.54^{(0.01)}$ & -5.45 & & & 0.50 & 0.25 & 0.25 & & \multicolumn{2}{|l|}{$64 \mathrm{G}, 73 \mathrm{~T}$} & positive (trend) \\
\hline
\end{tabular}




\section{EVAC4 amino acid alignment}

$\begin{array}{ll}\text { Mus musculus } & \text { MALMLVLFFLAAVLPPSLLQDNSQENSLEKLSTSKKSVQEEIVSKHNQLR } \\ \text { Mus domesticus } & \text { MALMLVLFFLAAVLPPSLLQDNSQENSLEKLSTSKKSVQEEIVSKHNQLR } \\ \text { Mus castaneus } & \text { MALMLVLFFLAAVLPPSLLQDNSQENSLEKLSTSKKSVQEEIVSKHNQLR } \\ \text { Mus spretus } & \text { MALMLVLFFLAAVLPPSLLQDNSQENSLEKLSTSKKSVQEEIVSKHNQLR } \\ & \\ \text { Mus musculus } & \text { RKVSPSGSDLLNMEWNYDAQVNAQQRADKCTFSHSPIELRTTNLKCGENL } \\ \text { Mus domesticus } & \text { RKVSPSGSDLLNMEWNYDAQVNAQQRADKCTFSHSPIELRTTNLKCGENL } \\ \text { Mus castaneus } & \text { RKVSPSGSDLLNMEWNYDAQVNAQQRADKCTFSHSPIELRTTNLKCGENL } \\ \text { Mus spretus } & \text { RKVSPSGSDLLNMEWNYDAQVNAQQRADKCTFSHSPIEIRTTNLKCGENL } \\ & \\ \text { Mus musculus } & \text { FMSSYLVPWSSVIQGWYNESKGLIFGVGPKQNVSVVGHHTQVVWKSNLQV } \\ \text { Mus domesticus } & \text { FMSSYLVPWSSVIQGWYNESKGLIFGVGPKQNVSVVGHHTQVVWKSNLQV } \\ \text { Mus castaneus } & \text { FMSSYLVPWSSVIQGWYNESKGLIFGVGPKQNVSVVGHHTQVVWKSNLQV } \\ \text { Mus spretus } & \text { FMSSYLAPWSSVIEGWYNEYKGLMFGVGRQNVSVVGHHTQVVWKSNLKV }\end{array}$

Mus musculus ACGVAECPENPLRYFYVCRYCPVLNYSGHYPSRPYLAYTARAPCASCPDR

Mus domesticus ACGVAECPENPLRYFYVCRYCP-----------------------

Mus castaneus ACGVAECPENPLRYFYVCRYCPVLNYSGHYPSRPYLAYTARAPCASCPDR

Mus spretus ACGVAECPENPLRYFYVCHYCPVSNYSGHYPSRPYLAYTARAPCASCPDR

$\begin{array}{ll}\text { Mus musculus } & \text { CEDGLCTKSCQYKDMSFWCKRLEYVCKHPGLKKRCLATCQC } \\ \text { Mus domesticus } & ---------------------1 \\ \text { Mus castaneus } & \text { CEDGLCTKSCQYKDMSFWCKRLEYVCKHPGLKKRCLATCQC } \\ \text { Mus spretus } & \text { CEDGLCTKSCQYKDMSFWCKRLTYVCKYPNLKKRCLATCQC }\end{array}$

Fig. 3 Amino acid alignment of Evac4 sequences. Sequence data gathered via NCBI nucleotide BLAST of genome sequences from wild-derived mouse strains carried out by the Sanger sequencing project (Yalcin et al. [46]). Amino acid substitutions marked in red

EVAC2 $\omega$ in mammals. Within clades, we found a trend for a positive correlation between relative testes mass and EVAC2 $\omega$ in Cetartiodactyla (Table 4). Correlations for the remaining clades were not significant. For Evac3a no significant correlations were found (Table 4).

\section{Discussion}

In this study we present an overview of selective pressures and tendencies of sexual selection-driven evolution of CRISP gene sequences. Moreover, due to differences in CRISP nomenclature between species we here propose a different set of gene names for use in evolutionary studies of CRISP (Fig. 1). Previous work has dealt with this inconsistency but a clear consensus of relationships between CRISP genes is not yet available [17, 44]. Based on our analysis, we propose that mouse Crisp1 (Evac3a) is an ortholog of human CRISP3 (EVAC3) that appears to have undergone rapid sequence divergence within the rodent clade after a putative duplication from Crisp2 (Evac2). We base this proposal on comparisons of sequence identities and gene tree clustering which show that human EVAC3 clusters more closely with mouse $E v a c 2$, the gene from which mouse $E v a c 3 a$ probably derived, rather than with mouse Evac3a. Additionally, rabbit $E V A C 3$ shows higher sequence identity with human EVAC3 than with mouse Evac3a. Human EVAC3 (human CRISP3) and mouse Evac4 (mouse Crisp3) have so far been assumed to be orthologs, mostly based on the fact that both genes show a wider range of expression compared to other EVAC genes. We could not find strong evidence for this. The sequence identity and clustering in the gene tree provide more evidence for a recent mouse-specific duplication event, with Evac4 deriving from Evac3a in mice. Previous studies have already shown CRISP3 (human EVAC3, mouse Evac4) to be ambiguous. Vadnais et al. [44] reported that the 


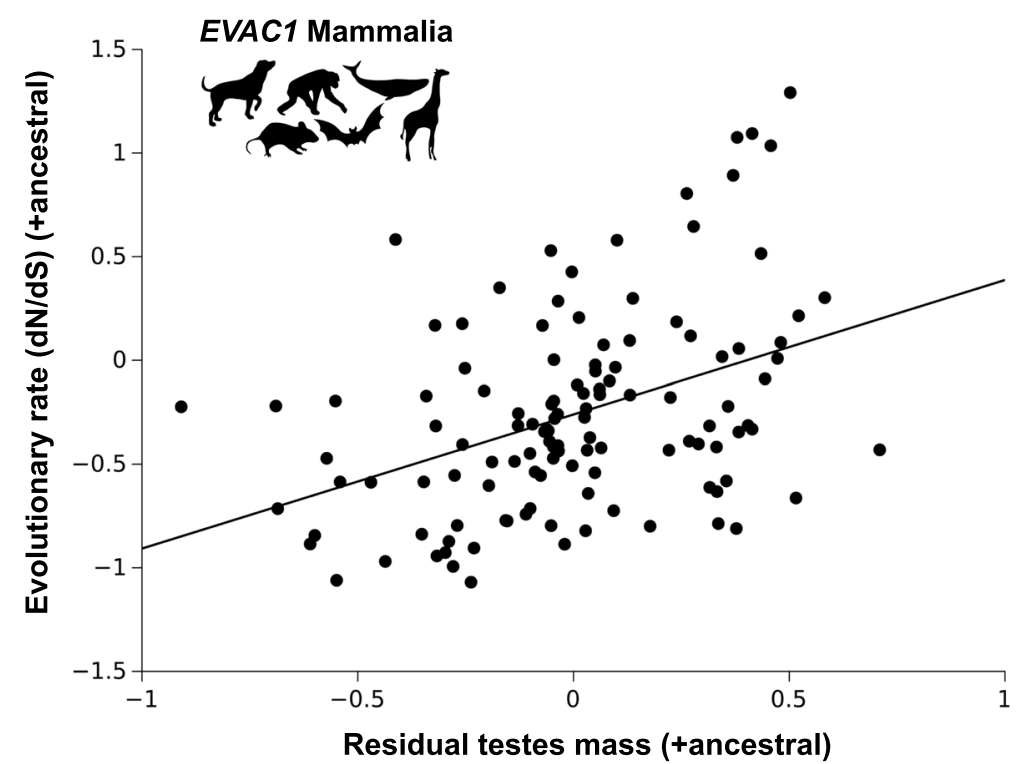

Fig. 4 Relationship between EVAC1 mammalian evolutionary rate (dN/dS) and residual testes mass including reconstructed ancestral node data. The association was examined by using COEVOL (see Table 4 for details)

GenBank sequence for pig CRISP3 was CRISP2. Pig CRISP3 has then been found within an unannotated region. CRISP3 sequence for pig, horse and cow cluster together but differ from human and mouse CRISP3 [44]. The diversity we see in the mammalian CRISP gene family and the difficulty resolving the orthologous relationships might be explained by rapid divergence of the gene cluster itself and, possibly, an increased susceptibility for duplication events. This certainly warrants further studies. The conclusions drawn here can only be considered preliminary and this analysis has been done for the sole purpose of gaining sufficient confidence in the understanding of interspecific relationships to undertake this comparative evolutionary study. A detailed analysis of the CRISP gene relationships and an adjustment of the nomenclature is necessary and is of great importance for future studies and to avoid wrong conclusions.

In this study we found $E V A C 1$ and $E V A C 2$, which are found across eutherian mammals, to be conserved in general, with signs of positive selection in primate EVAC1. Evac3a seems to evolve under a comparatively more relaxed constraint with positive selection on codon sites consistent with its proposed rapid divergence in the rodent clade. According to our findings, Evac4 seems to be specific to the genus Mus and shows signs of possible positive selection. Sexual selection seems to play a role in EVAC evolution and generally seems to favor an increase in evolutionary rate, although none of these trends have been found to be statistically significant.

Table 4 Results of COEVOL correlation analysis

\begin{tabular}{llllllll}
\hline & Clade & Variable 1 & Variable 2 & $\mathrm{n}$ & Covariances & Correlation coefficient & Posterior probability \\
\hline EVAC1 & Mammalia & $\boldsymbol{\omega}$ & relative testes mass & $\mathbf{3 9}$ & $\mathbf{2 . 0 6}$ & $\mathbf{0 . 4 1 6}$ & $\mathbf{0 . 0 7}$ \\
& Carnivora & $\omega$ & relative testes mass & 8 & -1.29 & -0.24 & 0.35 \\
& Cetartiodactyla & $\omega$ & relative testes mass & 9 & 2.29 & 0.45 & 0.17 \\
& Rodentia & $\boldsymbol{\omega}$ & relative testes mass & $\mathbf{6}$ & $\mathbf{3 . 5 3}$ & $\mathbf{0 . 6 5}$ & $\mathbf{0 . 0 9}$ \\
& Primates & $\omega$ & relative testes mass & 11 & 2.40 & 0.41 & 0.19 \\
EVAC2 & Mammalia & $\omega$ & relative testes mass & 39 & 1.21 & 0.35 & 0.14 \\
& Cetartiodactyla & $\boldsymbol{\omega}$ & relative testes mass & $\mathbf{1 0}$ & $\mathbf{0 . 7 2}$ & $\mathbf{0 . 6 7}$ & $\mathbf{0 . 0 6}$ \\
& Rodentia & $\omega$ & relative testes mass & 7 & -1.55 & -0.51 & 0.17 \\
& Primates & $\omega$ & relative testes mass & 10 & -0.16 & -0.11 & 0.40 \\
Evac3a & Rodentia & $\omega$ & relative testes mass & 6 & 0.06 & 0.09 & 0.58
\end{tabular}

Correlations with relative testes mass are corrected for body mass (see material and methods section), $\omega=$ evolutionary rate computed by COEVOL, $n=$ number of species in analysis, trends are shown in boldface 
EVAC proteins have been shown to be involved in various stages of the fertilization process, such as zona pellucida binding and gamete fusion [35]. Therefore, male expressed EVACs might show signs of coevolution with female specific interaction partners. In fact, previous studies have provided evidence for a possible co-evolution, and therefore interaction, between EVAC1 and EVAC2 and egg cell membrane protein CD9 in primates [42]. Signals of positive selection concentrated on specific regions of the gene sequence might thus be an indication of co-evolution with a binding partner. In the case of rodent Evac3a, there are positively selected sites found in the PR-1 (CAP) domain across rodents as well as in the CRD domain in several species. Positively selected sites in the PR-1 (CAP) domain of $E v a c 3 a$ are of specific interest here since it has been shown that this domain might be involved in gamete fusion [20], making it a very likely target for co-evolution with female binding partners. We also found positively selected sites in primate EVAC1, here located in the CRD region, which is likely to be involved in the regulation of ion channels such as CatSper [21, 38]. Adaptive evolution in its sequence might lead to a more efficient regulation or adjustment to different types of ion channels. An analysis of co-evolution between CRISP genes and possible (female) binding partners such as CD9, in a wider range of species would be of interest.

EVAC1 shows the strongest evidence for sexual selection-driven sequence evolution across mammals. $E V A C 1$ is expressed mainly in the epididymis and is involved in sperm capacitation and sperm-egg interaction in cooperation with other EVACs [35]. Interestingly, this gene seems to be the only EVAC not found in female reproductive tissues [48, 49] which may explain why a trend for a correlation with relative testes mass, a proxy for sperm competition, was found in this gene while not in the others. Genes acting in both male and female reproductive tissues might be subjected to different, even opposite pressures, due to sexual conflict, possibly obscuring any detectable signal of sexual selection during analysis. In genes confined to expression in male reproductive tissues, selective pressures due to sexual selection are more straightforward following only one direction, thus improving detection.

\section{Conclusions}

Even though EVAC1 and EVAC2 both seem to be conserved in general, which might be explained by their potential additional roles in other processes [17], positive selection can still be a factor in the evolution of these two genes. Positive selection might be detectable on lower taxonomic levels or might have happened in intervals during the genes evolutionary history. Similarly, the lack of a strong signal of sexual selection does not preclude a role for this selective force on EVAC sequence evolution. Selective pressures might not focus solely on gene sequence but may act on a larger scale, i.e., on regulatory sequences or favoring duplication events, as shown for Zonadhesin, a sperm ligand involved in sperm-egg interaction [50]. This might be especially true in mice which, so far, are the only mammals known to express four Crisp genes. Additionally, as shown in previous studies, sexual selection might be harder to detect across a wide range of clades since these pressures might affect taxa differently [14, 15]. This might be the case in $E V A C 2$ where we found signs of sexual selection acting only on cetartiodactylan sequences. An analysis testing for associations of regulatory sequences, epigenetic marks, number of gene duplication events, and transcript variants with levels of female promiscuity would be of great interest for this protein family. Although, the assignment of orthologs and the nomenclature need to be completely resolved in order to address future studies, we believe our observations contribute to a better understanding of CRISP family evolutionary history.

\section{Methods}

\section{Sequence data and phylogenetic tree}

Gene sequences of mammalian CRISPs (here called EVACs, as explained in Results and Discussion, and including the following: for $E V A C 1,61$ species; for $E V A C 2$, 65 species; for Evac3a, 8 rodent species; and for Evac4, 4 mouse species) were obtained from NCBI GenBank (Additional file 3), visualized with Geneious 5.5.9 (Biomatters, http://www.geneious.com/) and trimmed to coding sequences based on NCBI GenBank information. Sequences were manually checked to ensure correct trimming. Translation alignments were performed with PRANK [51] and subsequently stripped of columns containing gaps in more than $50 \%$ of the species to avoid bias due to ambiguously aligned regions [52]. PRANK is a phylogeny-aware progressive alignment especially applicable to analysis of selective pressures on coding sequences [53].

For EVAC1 and 2, in addition to alignments including all mammalian species (Additional files 4 and 5), we performed separate alignments for each mammalian clade studied (Primates, Rodentia, Chiroptera, Carnivora, Cetartiodactyla).

The phylogenetic trees of species included in this study were constructed as a consensus of phylogenies available from the literature (Additional files 6 and 7 and references therein).

Preliminary analysis of orthologous relationships between CRISP genes

We determined potential orthology based on gene sequence identity scores according to NCBI Blast [43], the 
existence or non-existence of specific CRISP orthologs in selected species, their genomic location and information by Nolan et al. [45] and Vadnais et al. [44]. Relationships were further investigated using PhylomeDB (http://phylomedb.org; 28-July-2017), a database of gene phylogenies providing information about the evolutionary history of genes by visualization of multiple sequence alignments and phylogenetic trees.

In addition to this, and using the method described above, we produced an alignment of all CRISP gene sequences included in this study, which was then used to calculate a CRISP gene tree. The gene tree was constructed using RAxML, implemented in Geneious 5.5.9 (Biomatters, http://www.geneious.com/), with 100 replicates of rapid bootstrapping.

\section{Analysis of selective pressures}

The nonsynonymous/synonymous substitutions rate ratio $(\omega, \mathrm{dN} / \mathrm{dS}$ or evolutionary rate) is an indicator of selective pressure at the protein level, with $\omega=1$ indicating neutral evolution, $\omega<1$ purifying selection, and $\omega>1$ diversifying positive selection [54]. To estimate gene sequence evolutionary rate across all mammals and additionally within mammalian clades, we used the application Codeml implemented in PAML $4[55,56]$. Codeml calculates the evolutionary rate based on different models. It takes as input a multiple sequence alignment and the corresponding phylogenetic tree. It then estimates evolutionary rates for the whole tree, each branch or branch groups, taking into account either the whole sequence, or each codon separately. The Codeml models applied are explained below. Likelihood-ratiotests (LRT) were performed to test if the alternative model presents a better fit to the dataset against the null model. For the Codeml codon frequency setting, as well as for the number of categories, we used the setting with the best fit for each analysis according to the preliminary likelihood-ratio-analysis.

\section{Evolutionary models applied in Codeml (PAML4) Branch analysis}

In order to obtain the evolutionary rates of mammalian clades, we computed the clade model comparing marked foreground branches (clade of interest) against the unmarked background in the analyzed phylogenetic tree. Three models were computed: M0 "one ratio" in which all branches were constrained to evolve at the same rate; MCfixed "two-ratio, foreground fixed" where the background $\omega$ was allowed to be estimated freely while the foreground $\omega$ was restrained to a value of $\omega=1$; and MC "two ratio" model which estimates for both the background and the foreground clade a free and independent $\omega$. To test if the foreground evolves at a significantly different rate than the background, we compared M0 versus MC by means of LRT. If foreground $\omega$ was significantly higher than 1 (LRT significant for MCfixed vs MC and $\omega>1$ ) we assumed positive selection acting on the foreground branches on whole sequence level. If foreground $\omega$ was significantly lower than 1 (LRT significant for MCfixed vs MC and $\omega>$ 1) we report purifying selection acting on the branch on whole sequence level. Relaxed selective constraint for the foreground branch is assumed if foreground evolves at a significantly different $\omega$ than the background (M0 vs MC), and this $\omega$ was not significantly different from 1 (MCfixed vs MC) [57]. P-values of LRTs were false discovery rate (FDR)-corrected.

\section{Branch-site analysis}

Similarly, two models were computed to test evolution along coding sequences and infer codons under positive selection for marked foreground branches (clade of interest) in contrast to the unmarked background. BSfixed "branchsite model A, foreground fixed" in which the codon site $\omega$ for background branches is allowed to be computed freely while the foreground is fixed and BS "branch-site model A" in which codon sites in both foreground and background are computed freely [58]. If LRT between BSfixed and BS is significant, and sites significantly belonging to the positive selected codon site (PSS) category are detected, we report positive selection on the detected codon sites for the clade of interest. P-values of LRTs were FDR-corrected.

\section{Site-analysis}

To apply a test for positive selection on codon sites across all branches, which is of interest in case of rodent Evac $3 a$, we applied a LRT comparing a null model that does not allow sites with $\omega>1$ with an alternative model that does. We applied two LRTs that have been widely used for this approach. The first compared model M1a "nearly neutral", which assumes values for $\omega$ between 0 and 1 , with model M2a "positive selection" which allows values of $\omega>1$. The second test compares two models assuming a $\beta$ distribution for $\omega$ values. In this case, the null model M7 that limits $\omega$ between 0 and 1 is compared to the alternative model M8, that adds an extra class of sites with an $\omega$ ratio estimated that can be greater than $1[59,60]$. We report positive selection on codon sites if LRT between both models is significant and sites significantly belonging to the positive selected site category are detected. If only one LRT is significant, we report a trend for the existence of PSS. Only sites significantly belonging to the positive selection site category in both alternative models are reported.

\section{Association between evolutionary rate and relative testes mass}

Sperm competition, evoked by females mating promiscuously, is a powerful selective force. An almost universal 
response to increased levels of sperm competition is an increase in testes size and sperm production [61]. The relationship between increased levels of sperm competition and larger relative testes mass has been widely demonstrated [62] and has been shown to associate to genetic paternity [63]. Thus, relative testes mass has been commonly used as a proxy for levels of sperm competition and female promiscuity. We here use relative testes mass as proxy for female promiscuity and increased selective pressure due to sexual selection in general since many CRISPs are sperm surface proteins and have the potential to be affected by sperm competition, cryptic female choice or male-female co-evolution. Data on both body and testes mass were obtained from the literature (Additional file 3). Residual testes mass data were obtained from a regression analysis including body mass as independent and testes mass as dependent variables, and used for graphical representation of multiple regression results.

To test for an association between evolutionary rate of $E V A C$ gene sequences and sexual selection, we employed the program COEVOL. COEVOL is a Bayesian Markov Chain Monte Carlo sampling software. This approach is used to test for correlation between genotype and phenotype data. It allows for a joint estimation of evolutionary rates for the input alignment and changes in the phenotypic input variables. Importantly, this software allows for detection of associations between genotypic and phenotypic data taking into account estimates of ancestral nodes, compared to previous approaches whereby the evolutionary rate was averaged from the root to the tip [47]. To test for sexual selection, correlations between testes mass and evolutionary rate were corrected for body mass by COEVOL using a multiple regression approach.

\section{Supplementary information}

Supplementary information accompanies this paper at https://doi.org/10. 1186/s12862-020-01632-5.

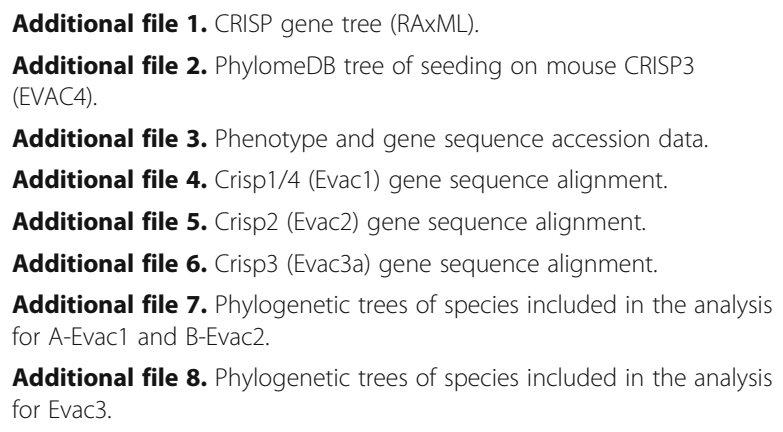

Additional file 8. Phylogenetic trees of species included in the analysis for Evac3.

\section{Acknowledgments}

We acknowledge support to cover the publication fee by the CSIC Open Access Publication Support Initiative through its Unit of Information Resources for Research (URICI).

\section{Authors' contributions}

LL: participated in the design of the study, compiled data, carried out evolutionary and statistical analysis and drafted the manuscript. ER, PC and NB: conceived the idea, participated in the design of the study and drafted the manuscript. All authors read and approved the final manuscript.

\section{Funding}

This work was supported by the Spanish Ministry of Economy, Industry and Competitiveness (grants CGL2011-26341 and CGL2016-80577-P).

\section{Availability of data and materials}

All data generated or analysed during this study are included in this published article. All gene sequences were taken from public databases (see details in Additional file 3). All phenotypic data (body mass and testes mass) were taken from the literature (Additional file 3).

Ethics approval and consent to participate

Not applicable.

\section{Consent for publication}

Not applicable.

\section{Competing interests}

The authors declare that they have no competing interests.

\section{Author details}

${ }^{1}$ Department of Biodiversity and Evolutionary Biology, Museo Nacional de Ciencias Naturales (CSIC), c/José Gutiérrez Abascal 2, 28006 Madrid, Spain. ${ }^{2}$ Institute of Pathology, Department of Developmental Pathology, University Hospital Bonn, Bonn 53127, Germany. Instituto de Biología y Medicina Experimental (IBYME-CONICET), C1428ADN Buenos Aires, Argentina.

Received: 16 June 2018 Accepted: 25 May 2020

Published online: 08 June 2020

\section{References}

1. Swanson WJ, Vacquier VD. The rapid evolution of reproductive proteins. Nat Rev Genet. 2002;3:137-44.

2. Turner LM, Hoekstra HE. Causes and consequences of the evolution of reproductive proteins. Int J Dev Biol. 2008;52:769-80.

3. Dorus S, Karr TL. Sperm proteomics and genomics. In: Birkhead TR, Hosken DJ, Pitnick S, editors. Sperm biology: an evolutionary perspective. Amsterdam: Elsevier; 2009. p. 435-69.

4. Findlay GD, Swanson WJ. Proteomics enhances evolutionary and functional analysis of reproductive proteins. BioEssays. 2010;32:26-36.

5. Vicens $A$, Lüke $L$, Roldan ER. Proteins involved in motility and sperm-egg interaction evolve more rapidly in mouse spermatozoa. PLoS One. 2014;9: e91302.

6. Birkhead TR, Pizzari T. Postcopulatory sexual selection. Nat Rev Genet. 2002; 3:262.

7. Dorus S, Evans PD, Wyckoff GJ, Choi SS, Lahn BT. Rate of molecular evolution of the seminal protein gene SEMG2 correlates with levels of female promiscuity. Nat Genet. 2004;36:1326-9.

8. Herlyn $H$, Zischler $H$. Sequence evolution of the sperm ligand zonadhesin correlates negatively with body weight dimorphism in primates. Evolution. 2007;61:289-98.

9. Ramm SA, Oliver PL, Ponting CP, Stockley P, Emes RD. Sexual selection and the adaptive evolution of mammalian ejaculate proteins. Mol Biol Evol. 2008;25:207.

10. Finn S, Civetta A. Sexual selection and the molecular evolution of ADAM proteins. J Mol Evol. 2010;71:231-40.

11. Prothmann A, Laube I, Dietz J, Roos C, Mengel K, Zischler H, Herlyn $\mathrm{H}$. Sexual size dimorphism predicts rates of sequence evolution of SPerm adhesion molecule 1 (SPAM1, also PH-20) in monkeys, but not in hominoid apes including humans. Mol Phylogenet Evol. 2012;63:52-63.

12. Walters JR, Harrison RG. Decoupling of rapid and adaptive evolution among seminal fluid proteins in Heliconius butterflies with divergent mating systems. Evolution. 2011;65:2855-71.

13. Lüke L, Vicens A, Serra F, Luque-Larena JJ, Dopazo H, Roldan ERS, Gomendio M. Sexual selection halts the relaxation of protamine 2 among rodents. PLoS One. 2011;6:e29247. 
14. Lüke $L$, Tourmente $M$, Roldan ERS. Sexual selection on protamine 1 in mammals. Mol Biol Evol. 2016a;3:174-84.

15. Lüke L, Tourmente M, Dopazo H, Serra F, Roldan ERS. Selective constraints on protamine 2 in primates and rodents. BMC Evol Biol. 2016b;16:21.

16. Dorus S, Wasbrough ER, Busby J, Wilkin EC, Karr TL. Sperm proteomics reveals intensified selection on mouse sperm membrane and acrosome genes. Mol Biol Evol. 2010;27:1235-46.

17. Gibbs GM, Roelants K, O'Brian MK. The CAP superfamily: cysteine-rich secretory proteins, antigen 5, and pathogenesis-related 1 proteins-roles in reproduction, cancer, and immune defense. Endocr Rev. 2008;29:865-97.

18. Yamazaki Y, Morita T. Structure and function of snake venom cysteine-rich secretory proteins. Toxicon. 2004;44:227-31.

19. Maeda T, Nishida J, Nakanishi Y. Expression pattern, subcellular localization and structure-function relationship of rat Tpx-1, a spermatogenic cell adhesion molecule responsible for association with Sertoli cells. Develop Growth Differ. 1999;41:715-22.

20. Ellerman DA, Cohen DJ, Da Ros VG, Morgenfeld MM, Busso D, Cuasnicú PS. Sperm protein "DE" mediates gamete fusion through an evolutionarily conserved site of the CRISP family. Dev Biol. 2006;297:228-37.

21. Gibbs GM, Scanlon MJ, Swarbrick J, Curtis S, Gallant E, Dulhunty AF, O'Bryan MK. The cysteine-rich secretory protein domain of Tpx-1 is related to ion channel toxins and regulates ryanodine receptor $\mathrm{Ca} 2+$ signaling. J Biol Chem. 2006;281:4156-63.

22. Abraham A, Chandler DE. Tracing the evolutionary history of the CAP superfamily of proteins using amino acid sequence homology and conservation of splice sites. J Mol Evol. 2017;85:137-57.

23. Cameo MS, Blaquier JA. Androgen-controlled specific proteins in rat epididymis. J Endocrinol. 1976;69:47-55.

24. Kasahara M, Figueroa F, Klein J. Random cloning of genes from mouse chromosome 17. Proc Natl Acad Sci U S A. 1987;84:3325-8.

25. Haendler B, Kratzschmar J, Theuring F, Schleuning WD. Transcripts for cysteine-rich secretory protein-1 (CRISP-1; DE/AEG) and the novel related CRISP-3 are expressed under androgen control in the mouse salivary gland. Endocrinology. 1993;133:192-8.

26. Jalkanen J, Huhtaniemi I, Poutanen M. Mouse cysteine-rich secretory protein 4 (CRISP4): a member of the CRISP family exclusively expressed in the epididymis in an androgen-dependent manner. Biol Reprod. 2005;72:1268-74.

27. Cohen DJ, Ellerman DA, Cuasnicú PS. Mammalian sperm-egg fusion: evidence that epididymal protein DE plays a role in mouse gamete fusion. Biol Reprod. 2000;1:462-8.

28. Busso D, Cohen DJ, Hayashi M, Kasahara M, Cuasnicu PS. Human testicular protein TPX1/CRISP-2: localization in spermatozoa, fate after capacitation and relevance for gamete interaction. Mol Hum Reprod. 2005;11:299-305.

29. Busso D, Cohen DJ, Maldera JA, Dematteis A, Cuasnicu PS. A novel function for CRISP1 in rodent fertilization: involvement in sperm—zona pellucida interaction. Biol Reprod. 2007;77:848-54.

30. Da Ros VG, Maldera JA, Willis WD, Cohen DJ, Goulding EH, Gelman DM, Rubinstein M, Eddy EM, Cuasnicu PS. Impaired sperm fertilizing ability in mice lacking cysteine-RIch secretory protein 1 (CRISP1). Dev Biol. 2008;1:12-8.

31. Brukman NG, Miyata $H$, Torres $P$, Lombardo D, Caramelo JJ, Ikawa M, Da Ros VG, Cuasnicú PS. Fertilization defects in sperm from cysteine-rich secretory protein 2 (Crisp2) knockout mice: implications for fertility disorders. Mol Hum Reprod. 2016;22:240-51.

32. Gibbs GM, Orta G, Reddy T, Koppers AJ, Martinez-Lopez P, de la VegaBeltran JL, Lo JC, Veldhuis N, Jamsai D, Mclntyre P, et al. 2011. Cysteine-rich secretory protein 4 is an inhibitor of transient receptor potential M8 with a role in establishing sperm function. Proc Natl Acad Sci U S A. 2011;108: 7034-9.

33. Turunen HT, Sipila P, Krutskikh A, Toivanen J, Mankonen H, Hamalainen V, Bjorkgren I, Huhtaniemi I, Poutanen M. 2012. Loss of cysteine-rich secretory protein 4 (Crisp4) leads to deficiency in sperm-zona pellucid interaction in mice. Biol Reprod. 2012;86:1-8.

34. Carvajal G, Brukman NG, Weigel Muñoz M, Battistone MA, Guazzone VA, Ikawa M. Haruhiko Miyata, Lustig L, Breton S, Cuasnicu PS. Impaired male fertility and abnormal epididymal epithelium differentiation in mice lacking CRISP1 and CRISP4. Sci Rep. 2018;8:17531.

35. Da Ros VG, Muñoz MW, Battistone MA, Brukman NG, Carvajal G, Curci L Gómez-Ellas MD, Cohen DB, Cuasnicu PS. From the epididymis to the egg: participation of CRISP proteins in mammalian fertilization. Asian J Androl. 2015;17:711-5.
36. Rochwerger L, Cohen DJ, Cuasnicu PS. Mammalian sperm-egg fusion: the rat egg has complementary sites for a sperm protein that mediates gamete fusion. Dev Biol. 1992;153:83-90.

37. Maldera JA, Weigel Muñoz M, Chirinos M, Busso D, Ge Raffo F, Battistone MA, Blaquier JA, Larrea F, Cuasnicu PS. Human fertilization: epididymal hCRISP1 mediates sperm-zona pellucida binding through its interaction with ZP3. Mol Hum Reprod. 2013;12:341-9.

38. Ernesto Jl, Muñoz MW, Battistone MA, Vasen G, Martínez-López P, Orta G, Figueiras-Fierro D, De la Vega-Beltran JL, Moreno IA, Guidobaldi HA, Giojalas L, Darszon A, Cohen DJ, Cuasnicú PS. CRISP1 as a novel CatSper regulator that modulates sperm motility and orientation during fertilization. J Cell Biol. 2015;210:1213-24.

39. Ren D, Navarro B, Perez G, Jackson AC, Hsu S, Shi Q, Tilly JL, Clapham DE. A sperm ion channel required for sperm motility and male fertility. Nature. 2001;413:603-9.

40. Carlson AE, Westenbroek RE, Quill T, Ren D, Clapham DE, Hille B, Garbers DL, Babcock DF. CatSper1 required for evoked Ca2+ entry and control of flagellar function in sperm. Proc Natl Acad Sci U S A. 2003; 100:14864-8.

41. Sunagar K, Johnson WE, O'Brien SJ, Vasconcelos V, Antunes A. Evolution of CRISPS associated with toxicoferan-reptilian venom and mammalian reproduction. Mol Biol Evol. 2012;29:1807-22.

42. Claw KG, George RD, Swanson WJ. Detecting coevolution in mammalian sperm-egg fusion proteins. Mol Reprod Dev. 2014;1:531-8.

43. Altschul SF, Gish W, Miller W, Myers EW, Lipman DJ. 1990. Basic local alignment search tool. J Mol Evol. 1990;215:403-10.

44. Vadnais ML, Foster DN, Roberts KP. Molecular cloning and expression of the CRISP family of proteins in the boar. Biol Reprod. 2008;79:1129-34.

45. Nolan MA, Wu L, Bang HJ, Jelinsky SA, Roberts KP, Turner TT, Kopf GS, Johnston DS. Identification of rat cysteine-rich secretory protein 4 (Crisp4) as the ortholog to human CRISP1 and mouse Crisp4. Biol Reprod. 2006;1: 984-91.

46. Yalcin B, Adams DJ, Flint J, Keane TM. Next-generation sequencing of experimental mouse strains. Mamm Genome. 2012;23:490-8.

47. Lartillot N, Poujol R. A phylogenetic model for investigating correlated evolution of substitution rates and continuous phenotypic characters. Mol Biol Evol. 2011;28:729-44.

48. Reddy T, Gibbs GM, Merriner DJ, Kerr JB, O'Bryan MK. Cysteine-rich secretory proteins are not exclusively expressed in the male reproductive tract. Dev Dyn. 2008;1:3313-23.

49. Evans J, D'Sylva R, Volpert M, Jamsai D, Merriner DJ, Nie G, Salamonsen LA, O'Bryan MK. Endometrial CRISP3 is regulated throughout the mouse estrous and human menstrual cycle and facilitates adhesion and proliferation of endometrial epithelial cells. Biol Reprod. 2015;92:99.

50. Herlyn H, Zischler $\mathrm{H}$. Tandem repetitive D domains of the sperm ligand zonadhesin evolve faster in the paralogue than in the orthologue comparison. J Mol Evol. 2006;63:602-11.

51. Löytynoja A, Goldman N. An algorithm for progressive multiple alignment of sequences with insertions. Proc Natl Acad Sci U S A. 2005;102:10557-62.

52. Talavera G, Castresana J. Improvement of phylogenies after removing divergent and ambiguously aligned blocks from protein sequence alignments. Syst Biol. 2007;56:564-77.

53. Löytynoja A, Goldman N. webPRANK: a phylogeny-aware multiple sequence aligner with interactive alignment browser. BMC Bioinformatics. 2010;11:579.

54. Goldman N, Yang Z. A codon-based model of nucleotide substitution for protein-coding DNA sequences. Mol Biol Evol. 1994;11:725-36.

55. Yang Z, Rannala B. Bayesian phylogenetic inference using DNA sequences, Markov chain Monte Carlo methods. Mol Biol Evol. 1997:14:717-24.

56. Yang Z. PAML 4, phylogenetic analysis by maximum likelihood. Mol Biol Evol. 2007;24:1586-91.

57. Yang Z. Likelihood ratio tests for detecting positive selection and application to primate lysozyme evolution. Mol Biol Evol. 1998;15:568-73.

58. Zhang J, Nielsen R, Yang Z. Evaluation of an improved branch-site likelihood method for detecting positive selection at the molecular level. Mol Biol Evol. 2005;22:2472-9.

59. Yang Z, Swanson WJ. Codon-substitution models to detect adaptive evolution that account for heterogeneous selective pressures among site classes. Mol Biol Evol. 2002;19:49.

60. Massingham T, Goldman N. Detecting amino acid sites under positive selection and purifying selection. Genetics. 2005;169:1753. 
61. Gomendio M, Harcourt H, Roldan ERS. Sperm competition in mammals. In: Birkhead TR, Møller AP, editors. Sperm competition and sexual selection. London: Academic Press; 1998. p. 667-751.

62. Hosken DJ, Ward PI. Experimental evidence for testis size evolution via sperm competition. Ecol Lett. 2001;22:10-3.

63. Soulsbury CD, Dornhaus A. Genetic patterns of paternity and testes size in mammals. PLoS One. 2010;5:103-8.

\section{Publisher's Note}

Springer Nature remains neutral with regard to jurisdictional claims in published maps and institutional affiliations.

Ready to submit your research? Choose BMC and benefit from:

- fast, convenient online submission

- thorough peer review by experienced researchers in your field

- rapid publication on acceptance

- support for research data, including large and complex data types

- gold Open Access which fosters wider collaboration and increased citations

- maximum visibility for your research: over $100 \mathrm{M}$ website views per year

At BMC, research is always in progress.

Learn more biomedcentral.com/submissions 